Rev. Biol. Trop., 47(1-2): 251-262, 1999

www.ucr.ac.cr www.ots.ac.cr www.ots.duke.edu

\title{
Mamíferos terrestres de la Reserva de la Biosfera de Sian Ka'an, Quintana Roo, México
}

Carmen Pozo de la Tijera y José Enrique Escobedo Cabrera

El Colegio de la Frontera Sur, Museo de Zoología, Apdo. Postal 424, 77000 Chetumal, Quintana Roo, México. Fax (983) 204-47, correo electrónico: cpozo@ nicte-ha.ecosur-qroo.mx

Recibido 3-IV-1998. Corregido 3-XII-1998. Aceptado 9-XII-1998

\begin{abstract}
Based on sampling at seven localities and an extensive bibliographic research, we present a species list of terrestrial mammals of the Sian Ka' an Biosphere Reserve, Quintana Roo, Mexico. During 57 days of fieldwork we used Sherman and Tomahawk traps, mist nets, rifles, collected data of trails and made direct observations. We recorded 70 species, eight orders, 22 families, and 57 genera. Six new records are added: Marmosa mexicana, Micronycteris microtis, Micronycteris schmidtorum, Eptesicus furinalis, Rhogeessa parvula, and Ototylomys phyllotis. Twelve species are listed as threatened following the Official Mexican Norm: Tamandua mexicana, Micronycteris brachyotis, Lonchorhina aurita, Alouatta pigra, Ateles geoffroyi, Herpailurus yagouaroundi, Leopardus pardalis, Leopardus wiedii, Panthera onca, Eira barbara, Potos flavus and Tapirus bairdii. Thirteen species are endemic to Mesoamerica: M. mexicana, T. mexicana, Mormoops megalophylla, Tonatia evotis, Bauerus dubiaquercus, A. pigra, A. geoffroyi, T. bairdii, Sciurus deppei, Sciurus yucatanensis, Heteromys gaumeri, O. phyllotis, Reithrodontomys gracilis and one endemic to Mexico: Peromyscus yucatanicus. We present tables of records for every locality and corresponding vegetation types for every species. Of 65 registered species, all were recorded in medium semievergreen forest, with $50 \%$ exclusively for this vegetation type. A reference collection was established with 200 specimens, representing 43 species.
\end{abstract}

Key words: Mammals, Quintana Roo, México, checklist, voucher collection, Biosphere Reserve, Sian Ka'an, new records.

La Reserva de la Biosfera de Sian Ka'an, al sureste en Quintana Roo, México, se extiende sobre una superficie de 534147 has, de las cuales el $40.5 \%$ son ambientes costeros marinos, $22.5 \%$ selvas medianas subperennifolias (SMS), $18 \%$ marismas con zacates (incluye sabanas), $13 \%$ de distintos tipos de manglar, 3\% vegetación perturbada, $2.5 \%$ selva baja inundable (SBI) y $0.5 \%$ de dunas costeras (Olmsted et al. 1983).

No existen registros de estudios sistemáticos de los mamíferos que habitan esta Reserva. Hay seis trabajos sobre mastozoología en los que se menciona como localidad algún sitio dentro de la Reserva. Gaumer (1917) registra
Spilogale putorius (Linnaeus, 1758), Eira barbara (Linnaeus, 1758), Odocoileus virginianus (Zimmermann, 1780) y Sciurus yucatanensis J. A. Allen, 1877. Genoways \& Jones (1975) citan a Gaumer (1917) mencionando sus registros. Más tarde, como parte de los trabajos para la creación de la Reserva, García (1983) publica una lista preliminar de vertebrados de Sian Ka'an, donde registra 26 especies de mamíferos terrestres sin mencionar las localidades de registro. Navarro-López et al. (1990), citan las localidades de Vigía Chico, El Ramonal, 6 y $8 \mathrm{~km}$ al "nornoreste" de Felipe Carrillo Puerto y Sabana del 11, agregando seis especies de roedores y a Didelphis virginiana Kerr, 1758 
como parte de la mastofauna de Sian Ka'an. Juárez \& Merediz (1994) registran 30 especies de murciélagos para el ambiente de SMS dentro de la Reserva sin mencionar las localidades de muestreo, por último, Merediz \& Ramírez (1994) registran la presencia de Tapirus bairdii (Gill, 1865), en el camino Chumpón-Vigía Chico, especie registrada desde 1983 por García. En total se conocían 64 especies de mamíferos terrestres para la Reserva al inició de este estudio.

Este trabajo es el primer muestreo sistemático y con una colección de referencia de los mamíferos de esta Reserva. La lista de especies de mamíferos registrada aquí, es una base sólida para estudios futuros de monitoreo en la Reserva.

\section{MATERIALES Y MÉTODOS}

La Reserva de la Biosfera de Sian Ka'an se localiza en la porción centro-oriente de la Peninsula de Yucatán, en el estado de Quintana Roo $\left(19^{\circ} 05^{\prime}-20^{\circ} 08^{\prime} \mathrm{N}, 87^{\circ} 23^{\prime}-88^{\circ} 03^{\prime}\right.$ $\mathrm{W})$. Se eligieron siete localidades con la finalidad de cubrir puntos de tierra firme en la parte norte, sur, zona de costa y tierra adentro (ver localidades), la parte central está cubierta principalmente por marismas (Fig. 1). En total se efectuaron once salidas de campo con un promedio de cinco días de recolecta, durante los meses de enero a agosto de 1995, noviembre de 1995 y de enero a marzo de 1996, completando 57 días de muestreo con la participación de cuatro personas por salida.

Los registros son de dos tipos: directos (recolectas de especímenes y observaciones de animales de fácil identificación) e indirectos (rastros). Para la captura de pequeños mamíferos se usaron 100 trampas Sherman por noche en cinco transectos de 20 trampas a una distancia de $10 \mathrm{~m}$ entre cada una y $50 \mathrm{~m}$ entre transectos, con cebo de avena y vainilla. En el caso de mamíferos de talla mediana, utilizamos tres trampas Tomahawk con cebo de sardina. Para los murciélagos se mantuvieron abiertas durante cinco horas cada noche, seis redes de niebla
( 2 x $12 \mathrm{~m}$ ) colocadas sobre caminos, cuerpos de agua y entre la vegetación, y para la captura de mamíferos de talla grande y algunos de talla mediana, se usaron escopetas calibre .16 y .20.

La identificación de los ejemplares fue por medio de guías de campo y claves especializadas (Hall 1981, Alvarez et al. 1994, Medellín et al. 1997). Las técnicas de preparación y rotulación de los ejemplares fueron las convencionales o estándares para colecciones de tipo científico (Hall 1981 y Ramírez-Pulido et al. 1989). El ordenamiento filogenético y la nomenclatura utilizada se hizo siguiendo las recomendaciones de Ramírez-Pulido et al. (1996) y para el caso del género Micronycteris la nomenclatura se hizo con base en Simmons (1996).

Los ejemplares fueron depositados en la colección mastozoológica del Museo de Zoología (ECO) de "El Colegio de la Frontera Sur" (ECOSUR), con números de catálogo $\mathrm{CH}-$ M0953 al CH-M1153, la información se incluyó en la base de datos de dicho museo, en el programa ACCESS 2.0.

Para el análisis de los datos se elaboró un Cuadro con la lista de las especies presentes en la Reserva donde se anotó si son endémicas (Flores \& Gerez 1994) y su estatus ecológico (de las que lo tienen), de acuerdo a CITES (Anónimo 1995), el Libro Rojo de la IUCN (Thornback \& Jenkins 1982) y la Norma Oficial Mexicana (NOM) (Anónimo 1994); en esta lista se distinguen los nuevos registros de especies. Se presenta la gráfica de representación comparativa de especies por familia y en Cuadros los datos de distribución ecológica y riqueza específica por localidad, con el número de individuos registrados por especie en cada una de ellas.

Localidades: Las localidades muestreadas y algunas de sus características (López-Ornat \& Consejo 1987 y obs. pers.), se detallan a continuación (Fig.1):

A) $49 \mathrm{~km}$ Felipe Carrillo Puerto-Vigía Chico. $19^{\circ} 47^{\prime} 55.3^{\prime \prime} \mathrm{N}$ y $87^{\circ} 40^{\prime} 34.4^{\prime \prime} \mathrm{W}$. SBI con una altura máxima de $14 \mathrm{~m}$, especies dominantes Metopium brownei, Manilkara zapota, Bucida buceras, Bucida spinosa, Lysiloma 


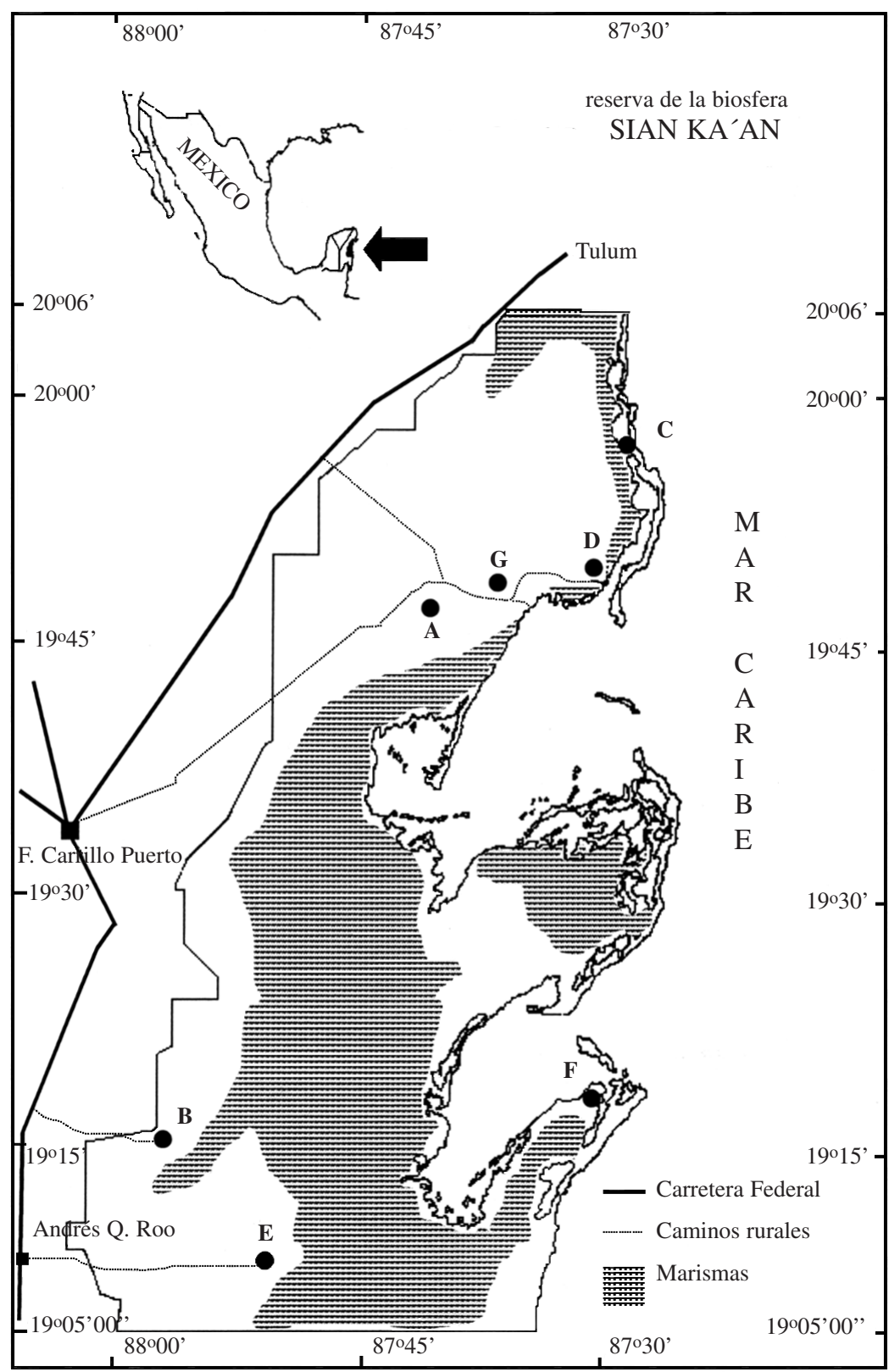

Fig. 1. Area de estudio con la posición de las siete localidades de muestreo (ver texto). 49 km Felipe Carrillo Puerto-Vigía Chico (A); 3 km S del crucero a X-Hazil, 15 km E (B); 6 km S de Boca Paila (C); 32 km Chumpón-La Glorieta (D); 42 km E de Andrés Quintana Roo (E); 8 km O de Punta Herrero (F); 27 km Chumpón-La Glorieta (G).

latisiqua, Dalbergia glabra, Crescentia cujete. Suelo somero (profundidad máxima $10 \mathrm{~cm}$ ).

B) $3 \mathrm{~km} \mathrm{~S}$ del crucero a X-Hazil, $15 \mathrm{~km} \mathrm{E}$ por brecha (PEMEX). $19^{\circ} 16^{\prime} 56.6 " \mathrm{~N}$ y $87^{\circ}$ 57 '35.1"W. SMS con altura máxima de $24 \mathrm{~m}$, especies dominantes $M$. brownei, M. zapota, Bursera simaruba, L. latisiqua, Thrinax radiata, Coccothrinax readii, Caesalpinia gaumeri, Nectandra coriacea. Suelo húmico (profundidad poco mayor a $10 \mathrm{~cm}$ ). 
C) $6 \mathrm{~km} \mathrm{~S}$ de Boca Paila. 19 59' 31.7’ N y $87^{\circ} 28^{\prime} 16.2^{\prime \prime} \mathrm{W}$. Ecotono Duna costera-manglar (altura variable), principales componentes $T$. radiata, Tournefortia gnaphalodes, Suriana maritima, Ambrosia hispida, Rhizophora mangle, Languncularia racemosa, Cordia sebestana, Hymenocallix littoralis, Sesuvium portulacastrum, Coccoloba cozumelensis. Suelo arenoso.

D) $32 \mathrm{~km}$ Chumpón-La Glorieta. 19² 49' $04.9^{\prime \prime} \mathrm{N}$ y $87^{\circ} 34^{\prime} 50^{\prime}$ 'W. SMS con altura máxima de $20 \mathrm{~m}$, especies dominantes $M$. zapota, M. brownei, B. simuraba, L. latisiqua, T. radiata, C. readii, Nectandra coriacea, Pseudophoenix sargentii. Suelo húmico (profundidad poco mayor a $10 \mathrm{~cm}$ ).

E) $42 \mathrm{~km}$ E de Andrés Quintana Roo. 19 09' $35^{\prime \prime}$ N y $87^{\circ} 53^{\prime} 20^{\prime \prime}$ W. SMS con altura máxima de $24 \mathrm{~m}$, especies dominantes $M$. brownei, M. zapota, B. simaruba, L. latisiqua, T. radiata, $C$. readii, $P$. sargentii, $C$. gaumeri, N. coriacea. Suelo húmico (profundidad poco mayor a $10 \mathrm{~cm}$ ). Cercana a esta localidad (300$500 \mathrm{~m} \mathrm{E}$ ) se encuentra vegetación de sabana y manglar.

F) $8 \mathrm{~km} \mathrm{O}$ de Punta Herrero. 19॰ 18'00.6" $\mathrm{N}$ y $87^{\circ} 32$ ' $51^{\prime \prime} \mathrm{W}$. SBI-manglar (altura máxima $14 \mathrm{~m}$ ), principales componentes M. zapota, $M$. brownei, $L$. racemosa, $R$. mangle, $P$. sargentii. Suelo húmico con arena.

G) $27 \mathrm{~km}$ Chumpón-La Glorieta. 19 $37^{\circ}$ $00^{\prime \prime} \mathrm{N}$ y $87^{\circ} 47^{\prime} 00^{\prime}$ 'W. SMS con altura máxima de $20 \mathrm{~m}$, especies dominantes M. Zapota, M. brownei, B. simaruba, L. latisiqua, T. radiata, $C$. readii, $N$. coriacea, $P$. sargentii. Suelo húmico (profundidad poco mayor a $10 \mathrm{~cm}$ ).

Debido a que la tierra firme de la Reserva (41.5\%) presenta diferente proporción de tipos de vegetación, el número de localidades para cada uno de estos tipos fue el siguiente: cuatro localidades con SMS, tres con manglar, dos con SBI, una con duna costera y una de sabana con baja proporción dentro de los marismas y de difícil acceso. Todas las localidades se muestrearon al menos durante seis días, excepto la localidad B y D que se muestrearon 18 y 12 días respectivamente; esto se debió también a la proporción de superficie ocupada por la SMS y a que las especies registradas en la literatura pa- ra la Reserva, son en su mayoría propias de este tipo de vegetación. En total se muestreó 41 días en SMS, 12 días en manglar, 11 días en SBI, 6 en duna costera y 6 en sabana.

\section{RESULTADOS}

Riqueza específica: Se obtuvo 819 registros, de los cuales 553 corresponden a individuos capturados y liberados, 66 a registros por métodos indirectos y por observación directa, 198 a individuos recolectados e ingresados en la colección científica (ECO) y en su correspondiente base de datos y dos registros de ejemplares depositados anteriormente en esta colección.

Se registraron 46 especies que junto con las registradas en la literatura para la Reserva, suman 70 especies de mamíferos terrestres (Cuadro 1), correspondientes a ocho órdenes, 22 familias y 57 géneros. En este estudio encontramos seis especies no registradas anteriormente (Cuadro 1), lo que representa un incremento del 9\%. El 17\%, del total de especies para la Reserva, está incluido dentro de la lista de especies protegidas por la NOM de las cuales, ocho especies están en peligro de extinción, dos especies amenazadas y dos más están catalogadas como raras (Anónimo 1994). La Reserva protege diez especies registradas por CITES (Anónimo 1995) y ocho especies registradas en el Libro Rojo de la IUCN (Thornback \& Jenkins 1982). Por otra parte, el $50 \%$ de las 28 especies endémicas de Mesoamérica y registradas para México (Flores-Villela \& Gerez 1994), están presentes en la Reserva, de las cuales una es endémica de México (Cuadro 1).

La Reserva posee el $76 \%$ de las familias de mamíferos terrestres conocidos para el estado de Quintana Roo (29), de las familias presentes en la Reserva el 68\% estan representadas en un $100 \%$ (Fig. 2), de éstas, la familia Phyllostomidae contiene el mayor número de especies (20).

En el Cuadro 2 se muestra en primer término, la riqueza específica presente en cada localidad con seis días de recolecta en cada una 


\section{CUADRO 1}

Lista de las especies presentes en la Reserva, donde se anotan los nuevos registros ( ), el estatus ecológico según CITES (Anónimo 1995), IUCN (Thornback \& Jenkins 1982) y NOM (Anónimo 1994), asi como las especies endémicas (E)

(Flores \& Gerez 1994).*

\begin{tabular}{|c|c|c|c|c|c|c|}
\hline FAMILIA & ESPECIE & CITES & IUCN & NOM & $\mathrm{E}$ & M \\
\hline Marmosidae & Marmosa mexicana Merriam, 1897 & & & & $\star$ & $\mathrm{a}$ \\
\hline \multirow[t]{2}{*}{ Didelphidae } & Didelphis marsupialis Linnaeus, 1758 & & & & & \\
\hline & Didelphis virginiana Kerr, 1758 & & & & & a \\
\hline Dasypodidae & Dasypus novemcinctus Linnaeus, 1758 & & & & & \\
\hline Myrmecophagidae & Tamandua mexicana (Saussure, 1860) & & & A & $\star$ & $\mathrm{b}, \mathrm{c}$ \\
\hline \multirow[t]{2}{*}{ Emballonuridae } & Peropteryx macrotis (Wagner, 1843) & & & & & $\mathrm{a}$ \\
\hline & Saccopteryx bilineata (Temminck, 1838) & & & & & a \\
\hline \multirow[t]{4}{*}{ Mormoopidae } & Mormoops megalophylla Peters, 1864 & & & & $\star$ & a \\
\hline & Pteronotus davyi Gray, 1838 & & & & & \\
\hline & Pteronotus parnellii (Gray, 1843) & & & & & a \\
\hline & Pteronotus personatus (Wagner, 1843) & & & & & \\
\hline \multirow[t]{20}{*}{ Phyllostomidae } & Micronycteris brachyotis (Dobson ,1879) & & & $\mathrm{P}$ & & a \\
\hline & Micronycteris microtis Miller, 1898 & & & & & a \\
\hline & Micronycteris schmidtorum Sanborn, 1835 & & & & & a \\
\hline & Desmodus rotundus (E. Geoffroy St.-Hilaire, 1810) & & & & & a \\
\hline & Diphylla ecaudata Spix, 1823 & & & & & \\
\hline & Trachops cirrhosus (Spix, 1823) & & & & & \\
\hline & Lonchorhina aurita Tomes, 1863 & & & $\mathrm{R}$ & & $\mathrm{a}$ \\
\hline & Tonatia brasiliense (Peters, 1866) & & & & & \\
\hline & Tonatia evotis Davis y Carter, 1978 & & & & $\star$ & \\
\hline & Glossophaga soricina (Pallas, 1766) & & & & & a \\
\hline & Artibeus intermedius J. A. Allen, 1897 & & & & & a \\
\hline & Artibeus jamaicensis Leach, 1821 & & & & & a \\
\hline & Artibeus lituratus (Olfers, 1818) & & & & & a \\
\hline & Carollia brevicauda (Schinz, 1821) & & & & & a \\
\hline & Carollia perspicillata (Linnaeus, 1758) & & & & & a \\
\hline & Centurio senex Gray, 1842 & & & & & a \\
\hline & Chiroderma villosum Peters, 1860 & & & & & a \\
\hline & Dermanura phaeotis Miller, 1902 & & & & & a \\
\hline & Sturnira lilium (E. Geoffroy St. -Hilaire, 1810) & & & & & a \\
\hline & Uroderma bilobatum Peters, 1866 & & & & & \\
\hline Natalidae & Natalus stramineus Gray, 1843 & & & & & a \\
\hline \multirow[t]{7}{*}{ Vespertilionidae } & Bauerus dubiaquercus (Van Gelder, 1959) & & & & $\star$ & a \\
\hline & Eptesicus furinalis (D’Orbigny, 1847) & & & & & a \\
\hline & Lasiurus ega (Gervais, 1856) & & & & & \\
\hline & Myotis keaysi J.A. Allen, 1914 & & & & & $\mathrm{a}$ \\
\hline & Rhogeessa aeneus Goodwin, 1958 & & & & & \\
\hline & Rhogeessa parvula H. Allen, 1866 & & & & & a \\
\hline & Rhogeessa tumida H. Allen, 1866 & & & & & a \\
\hline \multirow[t]{2}{*}{ Cebidae } & Alouatta pigra Lawrence, 1933 & I & $\mathrm{i}$ & $\mathrm{P}$ & $\star$ & \\
\hline & Ateles geoffroyi Kuhl, 1820 & I & $\mathrm{V}$ & $\mathrm{P}$ & $\star$ & $\mathrm{b}$ \\
\hline Canidae & Urocyon cinereoargenteus (Schreber, 1775) & & & & & a \\
\hline \multirow[t]{5}{*}{ Felidae } & Herpailurus yagouaroundi (Lacépède, 1809) & I & $\mathrm{i}$ & A & & $\mathrm{c}$ \\
\hline & Leopardus pardalis (Linnaeus, 1758) & I & $\mathrm{V}$ & $\mathrm{P}$ & & \\
\hline & Leopardus wiedii (Schinz, 1821) & I & $\mathrm{V}$ & $\mathrm{P}$ & & \\
\hline & Puma concolor (Linnaeus, 1771) & I & $\mathrm{P}$ & & & $\mathrm{b}, \mathrm{c}$ \\
\hline & Panthera onca (Linnaeus, 1758) & I & $\mathrm{V}$ & $\mathrm{P}$ & & $\mathrm{c}$ \\
\hline \multirow[t]{4}{*}{ Mustelidae } & Conepatus semistriatus (Boddaert, 1784) & & & & & $\mathrm{c}$ \\
\hline & Spilogale putorius (Linnaeus, 1758) & & & & & \\
\hline & Eira barbara (Linnaeus, 1758) & & & $\mathrm{P}$ & & $\mathrm{a}$ \\
\hline & Mustela frenata Lichtenstein, 1831 & & & & & \\
\hline \multirow[t]{3}{*}{ Procyonidae } & Potos flavus (Schreber, 1774) & & & $\mathrm{R}$ & & $\mathrm{a}$ \\
\hline & Nasua narica (Linnaeus, 1766) & & & & & $\mathrm{a}$ \\
\hline & Procyon lotor (Linnaeus, 1758) & & & & & $\mathrm{a}$ \\
\hline
\end{tabular}


(Cuadro 1: Continuación)

\begin{tabular}{|c|c|c|c|c|c|c|}
\hline FAMILIA & ESPECIE & CITES & IUCN & NOM & $\mathrm{E}$ & M \\
\hline Tapiridae & Tapirus bairdii (Gill, 1865) & I & $\mathrm{v}$ & $\mathrm{P}$ & $\star$ & $\mathrm{b}, \mathrm{c}$ \\
\hline \multirow{2}{*}{ Tayassuidae } & Pecari tajacu (Linnaeus, 1758) & II & & & & a \\
\hline & Tayassu pecari (Link, 1795) & II & & & & $\mathrm{b}, \mathrm{c}$ \\
\hline \multirow[t]{2}{*}{ Cervidae } & Mazama americana (Erxleben, 1777) & & & & & $\mathrm{b}, \mathrm{c}$ \\
\hline & Odocoileus virginianus (Zimmermann, 1780) & & & & & a \\
\hline \multirow[t]{2}{*}{ Sciuridae } & Sciurus deppei Peters, 1863 & & & & $\star$ & a \\
\hline & Sciurus yucatanensis J.A. Allen, 1877 & & & & $\star$ & a \\
\hline Heteromyidae & Heteromys gaumeri J.A. Allen y Chapman, 1897 & & & & $\star$ & a \\
\hline \multirow{6}{*}{ Muridae } & Mus musculus Linnaeus, 1758 & & & & & \\
\hline & Oryzomys couesi Alston, 1877 & & & & & \\
\hline & Ototylomys phyllotis Merriam, 1901 & & & & $\star$ & a \\
\hline & Peromyscus yucatanicus J.A. Allen y Chapman, 1897 & & & & $\boldsymbol{\Delta}$ & a \\
\hline & Reithrodontomys gracilis J.A. Allen y Chapman, 1897 & & & & $\bar{\star}$ & a \\
\hline & Sigmodon hispidus Say y Ord, 1825 & & & & & a \\
\hline Dasyproctidae & Dasyprocta punctata Gray, 1842 & & & & & $\mathrm{a}$ \\
\hline Agoutidae & Agouti paca (Linnaeus, 1766) & & & & & a \\
\hline
\end{tabular}

*I y II= apéndices cites, $\mathrm{i}=$ indefinida, $\mathrm{v}=$ vulnerable, $\mathrm{P}=$ en peligro de extinción, $\mathrm{A}=$ amenazada, $\mathrm{R}=$ rara, $\star$ = a mesoamérica, $\boldsymbol{\Lambda}=$ a México. $M=$ Este trabajo, $a=$ en colección de referencia, $b=$ observada y $c=$ rastros.

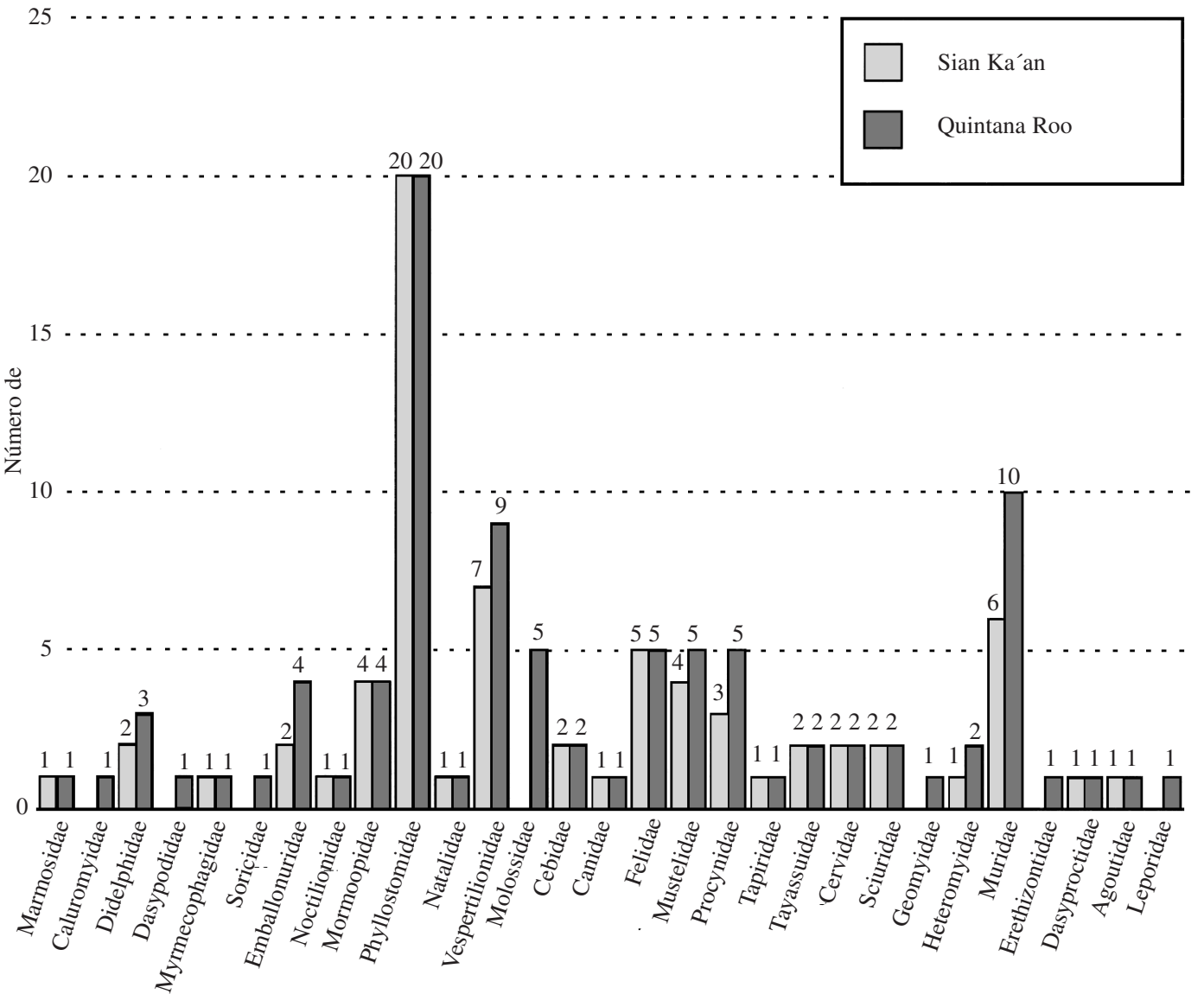

Fig. 2. Comparación del número de especies por familia encontrado en la RBSK, con respecto al registrado para el estado de Quintana Roo, México. 
de ellas y se encontró que las localidades con selva mediana subperennifolia (B, D, E, G) presentaron el mayor número de especies (>20) y que la localidad F presentó únicamente seis especies. Al aumentar el esfuerzo de recolecta para las localidades $\mathrm{B}$ y $\mathrm{D}$, se registraron por primera vez en este estudio, a Taman- dua mexicana (Saussure, 1860); Lonchorhina aurita Tomes, 1863; Desmodus rotundus (E. Geoffroy St.-Hilaire, 1810) y Herpailurus yagouaroundi (Lacépède, 1809) (Cuadro 2), además, el número de especies registradas para dichas localidades se incrementó, quedando 33 especies para la localidad B y 27 para D.

\section{CUADRO 2}

Riqueza de especies por localidad (con número de ejemplares recolectados), con un esfuerzo de seis días de recolecta

\begin{tabular}{|c|c|c|c|c|c|c|c|c|c|}
\hline ESPECIE & A & B & $\mathrm{C}$ & $\mathrm{D}$ & $\mathrm{E}$ & $\mathrm{F}$ & G & $\mathrm{B}^{*}$ & $\mathrm{D}^{*}$ \\
\hline Marmosa mexicana & & & & & & & 1 & & \\
\hline Didelphis virginiana & 1 & 1 & 1 & & 1 & & & & \\
\hline Tamandua mexicana & & & & & & & & 1 & \\
\hline Pteronotus parnellii & 5 & 2 & & & & & & & \\
\hline Micronycteris microtis & & & & & & & 1 & & \\
\hline Micronycteris schmidtorum & & & & & & & 2 & & \\
\hline Desmodus rotundus & & & & & & & & & 1 \\
\hline Lonchorhina aurita & & & & & & & & 1 & \\
\hline Glossophaga soricina & & & & & & & 1 & 1 & 10 \\
\hline Artibeus intermedius & 26 & 37 & 16 & 36 & 9 & 13 & 2 & & \\
\hline Artibeus jamaicensis & 9 & 17 & 23 & 37 & 3 & 15 & 1 & & \\
\hline Artibeus lituratus & & & 1 & & & & & & \\
\hline Carollia brevicauda & 1 & 1 & & & & & & & \\
\hline Carollia perspicillata & & & & 1 & 5 & & 1 & & \\
\hline Centurio senex & 1 & 2 & 2 & 1 & & & & & \\
\hline Chiroderma villosum & & & & 1 & & & & & \\
\hline Dermanura phaeotis & 22 & 39 & 7 & 14 & 7 & 4 & 4 & & \\
\hline Sturnira lilium & & & & & & 1 & & & \\
\hline Natalus stramineus & & & & 1 & & & & & \\
\hline Eptesicus furinalis & & & & 4 & & & & & \\
\hline Rhogeessa parvula & & & & & & & 1 & & \\
\hline Rhogeessa tumida & & 1 & & & & & & & \\
\hline Ateles geoffroyi & & 4 & & & 6 & & 5 & & \\
\hline Urocyon cinereoargenteus & 2 & 2 & 2 & 1 & 2 & & 1 & & \\
\hline Herpailurus yagouaroundi & & & & & & & & 1 & 1 \\
\hline Puma concolor & & 1 & & & 1 & & 1 & & \\
\hline Panthera onca & 1 & 1 & & 1 & 1 & & & & \\
\hline Conepatus semistriatus & 1 & & & & & & & 1 & \\
\hline Eira barbara & & & & 1 & 1 & & & 1 & \\
\hline Potos flavus & & 1 & & & 1 & & & & \\
\hline Nasua narica & & 1 & & 1 & 1 & & & & \\
\hline Procyon lotor & & & 8 & 1 & & 1 & & & \\
\hline Tapirus bairdii & 1 & 1 & & 1 & 1 & & 1 & & \\
\hline Pecari tajacu & 1 & 1 & & 1 & 1 & & 1 & & \\
\hline Tayassu pecari & & 1 & & 1 & 1 & & & & \\
\hline Mazama americana & 1 & 1 & & 1 & 2 & & 1 & & \\
\hline Odocoileus virginianus & 1 & 1 & & 1 & 1 & & 1 & & \\
\hline Sciurus deppei & & 1 & & & 1 & & 1 & & 4 \\
\hline Sciurus yucatanensis & 1 & & & 1 & 1 & & 1 & 2 & \\
\hline Heteromys gaumeri & & & & 3 & & 20 & 4 & 4 & \\
\hline Ototylomys phyllotis & 1 & 3 & & & 8 & & & & \\
\hline Peromyscus yucatanicus & & & & & 1 & & & 1 & \\
\hline Reithrodontomys gracilis & & & 3 & & & & 1 & & \\
\hline Sigmodon hispidus & & 1 & 3 & & 1 & & & & \\
\hline Dasyprocta punctata & & & & 1 & 1 & & 1 & 2 & \\
\hline Agouti paca & 1 & & & 1 & 1 & & 1 & 7 & \\
\hline RIQUEZA ESPECIFICA & 17 & 22 & 10 & 22 & 24 & 6 & 22 & +11 & +5 \\
\hline
\end{tabular}

* Para las localidades B y D se muestra el aumento en el número de especies registradas a causa de un incrementó en el esfuerzo de recolecta a 18 y 12 días respectivamente. 
Afinidad ecológica: Se registraron 65 especies presentes en la SMS, el 50\% de ellas se registraron como exclusivas a esta comunidad; le sigue la SBI con 26 especies (40\%) (Cuadro 3 ). En el caso del manglar (M) y los dos ecotonos (los cuales presentan asociación con manglar), se encontraron entre 10 y 12 especies (17\% prom.) y por último en la sabana $(\mathrm{S})$, que fue el tipo de vegetación mas pobre, se registran sólo cinco especies (8\%). Las especies que es- tán presentes en los cuatro tipos de vegetación son: Artibeus intermedius J.A. Allen, 1897; Artibeus jamaicensis Leach, 1821; Dermanura phaeotis Miller, 1902; Panthera onca (Linnaeus, 1758) y Procyon lotor (Linnaeus, 1758), las tres primeras presentaron el mayor número de individuos (Cuadros 2 y 3); en tres tipos de vegetación se encontraron Urocyon cinereoargenteus (Schreber, 1775) y Agouti paca (Linnaeus, 1766), que no se registraron en la sabana.

CUADRO 3

Distribución ecológica de los mamíferos de la Reserva de la Biosfera de Sian Ka’an.*

\section{ESPECIE}

Marmosa mexicana Didelphis virginiana Tamandua mexicana Peropteryx macrotis Saccopteryx bilineata Mormoops megalophylla

Pteronotus davyi

Pteronotus parnellii

Pteronotus personatus

Micronycteris brachyotis

Micronycteris microtis

Micronycteris schmidtorum

Desmodus rotundus

Diphylla ecaudata

Trachops cirrhosus

Lonchorhina aurita

Tonatia brasiliense

Tonatia evotis

Glossophaga soricina

Artibeus intermedius

Artibeus jamaicensis

Artibeus lituratus

Carollia brevicauda

Carollia perspicillata

Centurio senex

Chiroderma villosum

Dermanura phaeotis

Sturnira lilium

Uroderma bilobatum

Natalus stramineus

Eptesicus furinalis

Myotis keaysi

Lasiurus ega

Rhogeessa aeneus

Rhogeessa parvula

Rhogeessa tumida

Bauerus dubiaquercus

Alouatta pigra

Ateles geoffroyi

Urocyon cinereoargenteus

Herpailurus yagouaroundi

Leopardus pardalis

Leopardus wiedii

\begin{tabular}{|c|c|}
\hline SBI & $\underset{*}{\mathrm{SMS}}$ \\
\hline$*$ & $*$ \\
\hline \multirow[t]{5}{*}{$*$} & $*$ \\
\hline & $*$ \\
\hline & $*$ \\
\hline & $*$ \\
\hline & $*$ \\
\hline \multirow[t]{12}{*}{$*$} & $*$ \\
\hline & $*$ \\
\hline & $*$ \\
\hline & $*$ \\
\hline & $*$ \\
\hline & $*$ \\
\hline & $*$ \\
\hline & $*$ \\
\hline & $*$ \\
\hline & $*$ \\
\hline & $*$ \\
\hline & $*$ \\
\hline$*$ & $*$ \\
\hline \multirow[t]{2}{*}{$*$} & $*$ \\
\hline & $*$ \\
\hline \multirow[t]{2}{*}{$*$} & $*$ \\
\hline & $*$ \\
\hline$*$ & $*$ \\
\hline & $*$ \\
\hline \multirow[t]{12}{*}{$*$} & $*$ \\
\hline & $*$ \\
\hline & $*$ \\
\hline & $*$ \\
\hline & $*$ \\
\hline & $*$ \\
\hline & $*$ \\
\hline & $*$ \\
\hline & $*$ \\
\hline & $*$ \\
\hline & $*$ \\
\hline & $*$ \\
\hline$*$ & $*$ \\
\hline$*$ & $*$ \\
\hline \multirow[t]{3}{*}{$*$} & $*$ \\
\hline & * \\
\hline & $*$ \\
\hline
\end{tabular}

M

S

M-DC

ECOTONOS

$*$
$*$
$*$

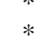

$*$
$*$
$*$

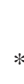

*

$*$

*

$*$
M-SBI

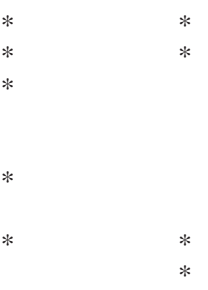

* 
(Cuadro 3: Continuación)

\begin{tabular}{|c|c|c|c|c|c|c|}
\hline ESPECIE & SBI & SMS & M & $\mathrm{S}$ & M-DC & M-SBI \\
\hline Puma concolor & $*$ & $*$ & & & & \\
\hline Panthera onca & * & * & $*$ & $*$ & & \\
\hline Conepatus semistriatus & * & * & & & & \\
\hline Eira barbara & * & * & & & & \\
\hline Potos flavus & & * & & & & \\
\hline Nasua narica & $*$ & $*$ & & & & \\
\hline Procyon lotor & * & * & $*$ & $*$ & $*$ & $*$ \\
\hline Tapirus bairdii & & & $*$ & $*$ & & \\
\hline Pecari tajacu & & & $*$ & $*$ & & \\
\hline Tayassu pecari & & & * & $*$ & & \\
\hline Mazama americana & & & $*$ & $*$ & & \\
\hline Odocoileus virginianus & $*$ & $*$ & & & & $*$ \\
\hline Sciurus deppei & & * & & & & \\
\hline Sciurus yucatanensis & & * & $*$ & & & \\
\hline Heteromys gaumeri & & $*$ & $*$ & & $*$ & $*$ \\
\hline Ototylomys phyllotis & & * & $*$ & & & \\
\hline Oryzomys couesi & & $*$ & & & & \\
\hline Peromyscus yиcatanicus & & * & & & & \\
\hline Reithrodontomys gracilis & & * & $*$ & & * & \\
\hline Sigmodon hispidus & & * & & & * & $*$ \\
\hline Dasyprocta punctata & $*$ & * & & & & \\
\hline Agouti paca & $*$ & $*$ & $*$ & & & $*$ \\
\hline TOTAL DE ESPECIES & 26 & 65 & 12 & 5 & 12 & 10 \\
\hline
\end{tabular}

* Se incluyen las especies que fueron registradas durante este estudio y las que bibliográficamente registran el tipo de vegetación. SBI= Selva baja inundable, SMS= Selva mediana subperennifolia, M= Manglar, S= Sabana, M-DC= Ecotono Manglar-Duna costera, M-SBI= Ecotono Manglar-Selva baja inundable.

Las especies de interés especial y encontradas en cinco localidades son S. yucatanensis y T. bairdii, esta última, no se encontró en las localidades con manglar o duna costera.

\section{DISCUSIÓN}

Los resultados resaltan la importancia ecológica que reviste esta Reserva para la protección de los mamíferos terrestres de México, en especial por las especies que se registraron y que están catalogadas en la NOM. La Reserva alberga $74 \%$ de las especies de mamíferos terrestres registradas para Quintana Roo (94) y el 16\% de los 450 mamíferos terrestres presentes en México (Cervantes et al. 1994). También su importancia reside en la presencia de especies endémicas, en este estudio fueron registradas nueve especies endémicas a Mesoamérica (Cuadro 1), y una endémica de México: Peromyscus yucatanicus J. A. Allen y Chapman, 1897. De las especies endémicas a Mesoamérica, T. mexicana ha sido catalogada co- mo amenazada por la NOM y en este trabajo la registramos sólo en una ocasión. La especie $T$. bairdii catalogada en la NOM como en peligro de extinción, como vulnerable por la IUCN y en el apéndice I del CITES, se detecta presente en todas las localidades con vegetación de selva (SMS y SBI), lo que nos permite suponer que presenta una amplia distribución dentro de la Reserva y con un aparente alto número poblacional. También se protegen a cinco de los seis felinos registrados para México y que son considerados en el Libro Rojo de la IUCN y en el apéndice I del CITES, lo que afirma la importancia de la Reserva en la protección de la mastofauna de interés especial a nivel nacional e internacional.

Algunas de las especies citadas en la literatura no fueron registradas durante el transcurso de este trabajo v. gr. Leopardus pardalis (Linnaeus, 1758); Leopardus wiedii (Schinz, 1821) y Didelphis marsupialis Linnaeus 1758, su presencia en localidades cercanas a la Reserva que tienen ambientes muy similares a los encontrados en ella, nos hace pensar que puedan estar 
presentes. En cuanto al registro de Alouatta pigra Lawrence, 1933 como parte de la fauna residente en la Reserva, recomendamos que sea confirmado debido a que sólo es citado por García (1983). No descartamos que pueda encontrarse en zonas poco accesibles como son los Petenes, pero reiteramos que debe documentarse su presencia dentro de la Reserva.

Los murciélagos es el grupo mejor representado con 34 especies, es decir con el 50\% de los mamíferos registrados para esta área; le siguen los carnívoros y los roedores. La composición taxonómica de la comunidad de mamíferos de la Reserva es rica y pensamos que puede ser incrementada si se continúa el estudio explorando otro tipo de ambientes presentes en la Reserva y que no fueron muestreados, como los Petenes y Tasistales.

Las especies A. intermedius, A. jamaicensis y $D$. phaeotis fueron registradas con altos números de individuos por especie en todos los tipos de vegetación, esto confirma que son especies con plasticidad ecológica (Medellin et al. 1992) y por lo tanto con un amplio rango de distribución que va desde Sinaloa, en el Pacífico Mexicano y el Golfo de México hasta el sur de Panamá (Hall 1981).

Para los nuevos registros se encontró un sólo individuo de Micronycteris microtis Miller, 1898; dos individuos de Marmosa mexicana Merriam, 1897; Micronycteris schmidtorum Sanborn, 1835 y Rhogeessa parvula H. Allen, 1866; cuatro de Eptesicus furinalis (D’Orbigny, 1847) y doce de Ototylomys phyllotis Merriam, 1901. Las seis especies se recolectaron en SMS. La distribución para cada especie se presenta a continuación:

$-M$. mexicana se distribuye desde Tamaulipas hasta el oeste de Panamá (Hall 1981, Gardner 1993). En 1990, Navarro et al., la registran para una localidad cercana a la Reserva como el primer registro para el estado de Quintana Roo. Localidad G. (No. de catálogo: ECO-CHM1094).

$-M$. microtis tiene un rango de distribución que abarca desde la Península de Yucatán y las vertientes del Golfo de México y del Pacífico hasta Perú, Bolivia y Brasil (Hall 1981, Koop- man 1993 y Nowak 1994). Sin embargo, su distribución al este de la Península de Yucatán, se basa en un sólo registro para Quintana Roo localizado en la Isla de Cozumel. El registro aqui reportado, es el primer registro continental para el estado. Localidad G. (No. de catálogo: ECO-CHM1088).

-M. schmidtorum es registrada para la Península de Yucatán y el sur de México hasta Venezuela, NE de Perú y NE de Brasil (Koopman 1993 y Nowak 1994), existe un registro dudoso de un ejemplar juvenil para la Isla de Cozumel (Jones et al. 1973), no existen registros en localidades cercanas a la Reserva; tanto M. microtis, como M. schmidtorum son especies poco comunes en la Península de Yucatán. Localidad G. (No. de catálogo: ECOCHM1093).

-E. furinalis se distribuye en ambas vertientes de México, desde las costas de Jalisco y Tamaulipas hasta el norte de Argentina (Koopman 1993). Para esta especie, tampoco existe registro en alguna localidad cercana a la Reserva. Localidad D. (No. de catálogo: ECOCHM1026).

$-R$. parvula. No obstante que Jones (1973) cita a esta especie como ampliamente distribuida en la Península de Yucatán pero con una abundancia poco común, otros autores (Hall 1981, Koopman 1993 y Nowak 1994), la proponen como una especie con distribución exclusiva para el Pacífico Mexicano; este registro confirma la distribución propuesta por Jones desde 1973. Cabe hacer notar que Jones menciona que esta especie no es capturada en redes, sin embargo, nosotros hemos capturado 6 ejemplares con redes de niebla. Localidades D y G. (No. de catálogo: ECO-CHM1077 y ECO-CHM1248).

-Por último, la especie O. phyllotis se distribuye en las costas de Costa Rica, hasta el norte de Yucatán, Tabasco y Chiapas. Existe un registro aislado de Guerrero (Hall 1981 y Musser \& Carleton 1993), es una especie abundante y ampliamente distribuida en la Península de Yucatán. Localidades A, B, D y E. (No. de catálogo: ECO-CHM0001, ECO-CHM0970 ECO-CHM0985 y ECO-CHM1019). 


\section{AGRADECIMIENTOS}

Este trabajo fue posible gracias al apoyo financiero de CONABIO (B114) y de ECOSUR. El INE autorizó el permiso de recolecta DOO 700(2).-01442. Queremos agradecer a nuestros compañeros de campo su apoyo para la realización de este estudio, en especial a Alejandro y Margarito Tuz Novelo. A Livia León Paniagua por su ayuda en la identificación de los ejemplares. A Carlos Galindo-Leal y otra vez a Livia León P. por la revisión del manuscrito.

\section{RESUMEN}

Con base en el muestreo de siete localidades y una extensa revisión bibliográfica, se obtuvo la lista de especies de mamíferos terrestres de la Reserva de la Biosfera de Sian Ka'an estado de Quintana Roo, México. Durante 57 días de campo, se utilizaron trampas Sherman, Tomahawk, redes de niebla y escopetas, se registraron rastros y observaciones directas. Se registraron 70 especies, ocho órdenes, 22 familias y 57 géneros. Se encontraron seis especies como nuevos registros: Marmosa mexicana, Micronycteris microtis, Micronycteris schmidtorum, Eptesicus furinalis, Rhogeessa parvula y Ototylomys phyllotis. Doce especies son catalogadas bajo algún riesgo ecológico según la Norma Oficial Mexicana; trece especies son endémicas a Mesoamérica y una endémica de México. Se presentan cuadros de abundancia relativa, registro por localidades y por tipo de vegetación de cada especie.

\section{REFERENCIAS}

Alvarez, T., S.T. Alvarez-Castañeda \& J.C. López-Vidal. 1994. Clave para murciélagos mexicanos. Centro de Investigaciones Biológicas del Noreste, S.C. y Escuela Nacional de Ciencias Biológicas, Instituto Politécnico Nacional. México. 64 p.

Anónimo. 1994. Proyecto de Norma Oficial Mexicana que determina las especies de flora y fauna silvestres terrestres y acuáticas, raras, endémicas, amenazadas, en peligro de extinción y sujetas a protección especial. Diario Oficial de la Federación. México. 488:30-37.

Anónimo. 1995. Appendices I, II, y III to the Conservation on International Trade in Endangered Species of wild Fauna and Flora., U.S. Department of the Interior and U.S.F.W.S. Washington. 23 p.
Cervantes, F.A., A. Castro-Campillo \& J. Ramírez-Pulido. 1994. Mamíferos terrestres nativos de México. An. Inst. Biol., Univ. Nal. Autón. México, Ser. Zool. 65:177-190.

Flores, O. \& P. Gerez. 1994. Biodiversidad y conservación en México: Vertebrados, vegetación y uso de suelo. Comisión Nacional para el Conocimiento y Uso de la Biodiversidad y Univ. Nal. Autón. México. México. $439 \mathrm{p}$.

García, M. 1983. Fauna silvestre, p. 105-111. In Anónimo (ed.). Sian Ka'an. Centro de Investigaciones de Quintana Roo y Secretaría de Desarrollo Urbano y Ecología. Puerto Morelos, México. 215 p.

Gardner, A.L. 1993. Order Didelphimorphia, p. 18. In D. E. Wilson \& D.M. Reeder (eds.) Mammal species of the world. A taxonomic and geographic reference. Smithsonian Institution Press, Washington, D.C. 1207 p.

Gaumer, G.F. 1917. Monografía de los mamíferos de Yucatán. Depto. Talleres gráficos, Secretaría de Fomento, México. XLI + 331 p.

Genoways, H.H. \& J.K. Jones, Jr. 1975. Annotated checklist of mammals of the Yucatan Peninsula, México. IV. Carnivora, Sirenia, Perissodactyla, Artiodactyla. Occas. Papers Mus., Texas Tech Univ. 26:1-22.

Hall, E.R. 1981. The Mammals of North America. The Ronald Press Co. Nueva York., Vol. 1:XXX+546+79, Vol. 2:VI+601-1181+90.

Jones, J.K., Jr., J.D. Smith \& H.H. Genoways. 1973. Annotated checklist of Mammals of the Yucatan Peninsula, México. I. Chiroptera. Occas. Papers Mus., Texas Tech Univ., 13:1-31.

Juárez, J. \& G. Merediz A. 1994. Lista sistemática de Quirópteros registrados para Sian Ka'an hasta 1994. Sian Ka'an. Serie documentos 2:61-62.

Koopman, K.F. 1993. Order Quiroptera, p. 137-241. In D.E. Wilson \& D.M. Reeder (eds.) Mammal species of the world. A taxonomic and geographic reference. Smithsonian Institution Press, Washington, D.C. 1207 p.

López-Ornat, A. \& J.J. Consejo. 1987. Sian Ka’an, reserva de la biosfera: Plan de manejo, edición preliminar. Secretaría de Desarrollo Urbano y Ecología. Chetumal, México. 141 p.

Medellín, R.A., O. Sánchez-Herrera \& G. Urbano-V. 1992. Ubicación zoogeográfica de la Selva Lacandona, Chiapas, México, a través de su fauna de quirópteros, p. 233-251. In Vásquez-Sánchez, M.A. \& M.A. Ramos (eds.). Reserva de la Biosfera Montes Azules, Selva Lacandona: Investigación para su conservación. Publ. esp. Ecósfera México. 1:233-251.

Medellín, R.A., H.T. Arita \& O. Sánchez H. 1997. Identificación de los Murciélagos de México. Clave de 
campo. Asociación Mexicana de Mastozoología A. C. Publ. esp. no. 2. México. 83 p.

Merediz, G. \& P.A. Ramírez. 1994. Registros de tapir (Tapirus bairdii) en Sian Ka'an y su zona de cooperación. Sian Ka'an. Serie documentos. No. 2:60-61.

Musser, G.G. \& M.D. Carleton. 1993. Family Muridae, p. 18. In D.E. Wilson \& D.M. Reeder (eds.) Mammal species of the world. A taxonomic and geographic reference. Smithsonian Institution Press, Washington, D.C. 1207 p.

Navarro-López, D., T. Jiménez \& J. Juárez. 1990. Los mamíferos de Quintana Roo, p. 371-450. In D. NavarroLópez \& J.G. Robinson (eds.). Diversidad Biológica de la Reserva de la Biosfera de Sian Ka'an Quintana Roo, México. Centro de Investigaciones de Quintana Roo. México. 471 p.

Nowak, R.M. 1994. Walker's Bats of the World. The Johns Hopkins University Press. Baltimore \& Londres. $287 \mathrm{p}$.
Olmsted, Y.C., A. López Ornat \& R. Durán García. 1983. Vegetación de Sian Ka'an. Reporte preliminar. p. 6384 In Anónimo (ed.). Sian Ka'an. Centro de Investigaciones de Quintana Roo y Secretaria de Desarrollo Urbano y Ecología. Puerto Morelos, México. 215 p.

Ramírez-Pulido, J., I. Lira, S. Gaona, C. Mudespcher \& A. Castro. 1989. Manejo y mantenimiento de Colecciones Mastozoológicas. Universidad Autónoma Metropolitana. México. 127 p.

Ramírez-Pulido, J., A. Castro-Campillo, J. Arroyo-Cabrales \& F.A. Cervantes. 1996. Lista taxonómica de los mamíferos terrestres de México. Occas. Papers Mus., Texas Tech Univ., No. 158. 62 p.

Simmons, N.B. 1996. A new species of Micronycteris (Chiroptera: Phyllostomidae) from northeastern Brazil, with comments on phylogenetic relationships. American Museum Novitates 3158:1-34.

Thornback J. \& M. Jenkins. 1982. The IUCN Mammal Red Data Book. Publ. IUCN. Reino Unido. 515 p. 